\title{
BIOLOGICAL PHENOMENA MIMIC ASTROPHYSICAL PRINCIPLES OF GRAVITATION - OPENING PERSPECTIVE
}

\author{
Dr. Iresh Ranjan Bhattacharjee
}

Principal Scientist, Institute for Intrinsic Gravitation Biology, Guwahati, India

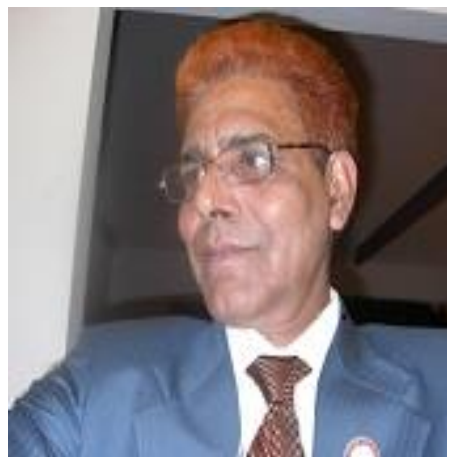

DR.IRESH RANJAN BATTACHARJEE

\section{Keywords:}

fluids, neutral buoyancy, star formation, macromolecular movements, ultracentrifugation,

\begin{abstract}
A B S T R A C T
It is conjectured that phenomena in biological microworld could be equated with astrophysical principles of gravitation. Fluids, gases that constitute 50-90\% of the total accredited biomass causes seclusion from stronger external gravitational fields. Seclusion due to buoyant condition is reflected in apparent 'weight' reduced. The gvalue (self) to the tune of nanometer per second square in a massive body of a planet may be negligible, but in an isolated living mass at picometer distance, such acceleration is quite a significant force. Homogeneous and heterogeneous accretion; heating and cooling phenomena as well as the rhythmic pattern of growth due to elastic collisions in massive star; heat transfer mechanisms viz. radiation, (perspiration), conduction and convection; site of human core body temperature at liver, kidney, heart and parts of brain at central region under initial circular fetus situation; or coldest part of the periphery at toe, foot, hand demonstrates similarity between biological and astrophysical words. Mechanical movement of macromolecules under neutral buoyant condition of the principle 'heavier molar mass plus higher the density-faster the central travel from periphery' for nucleic acid and protein; fats \& lipids in addition to molar mass and density-variation in temperature; distribution carbohydrates in protoplasm under miscible condition due to solubility under granular soil and gel properties were equated with movement phenomena under astrophysical principle. Centrifugation as standard protocol for separating organelles is considered as inversion or reversing the natural setting on losing its compression memory.
\end{abstract}

Citation: Iresh Ranjan Bhattacharjee (2018). Biological Phenomena Mimic Astrophysical Principles Of Gravitation - Opening Perspective. International Journal of Advanced Multidisciplinary Scientific Research (IJAMSR ) ISSN:2581-4281 Vol 1, Issue 6, August, 2018, \#Art.611, pp1-16

\section{Introduction}

The 'Self gravitation bio', a new topic has been annexed in the study of biophysics by the Biophysical Society (USA) in 2008 on being presented by the author $[1,2]$. It has also gain momentum after publication of series of articles, latest on 'Gravitation induced Mechanical movement in cells [3]. Self gravitation bio contemplates the concepts of gravity to the microscopic world of life 


\section{International Journal of Advanced Multidisciplinary Scientific Research (IJAMSR) ISSN:2581-4281}

science. Gravity as variable in life science was not proposed earlier. But many of its working remains elusive. Gravity is customarily considered as a long distance force acting on a massive body at macroscopic length scale and limited to astrophysical world. The presence of gravity at macroscopic scale at $10^{\wedge} 6$ to $10^{\wedge 36} \mathrm{~m}$, especially from planet to universe is virtually understood through various astrophysical principles. Similarly the presence of gravity at microscopic scale at $10^{\wedge}-12$ or so to $10^{\wedge}-36 \mathrm{~m}$ is being felt under particle physics mainly through quantum gravity. On mesoscopic length scale, interaction phenomena of mutual gravity are nowa-days getting some attention after initiation of space research on life sciences at microgravity. In contrast, biological micro world at mesoscopic scale $\left(10^{\wedge}\right.$ to $10^{\wedge}$ ${ }^{12} \mathrm{~m}$ ) is kept outside the purview of gravitation because of historical oversight. The recent detection of gravitational wave in realty might be an eye opener to the biologists. Under equal quantity of gravitational mass, the distinction between living and non-living is never projected in proper gravitational perspective. In living biomass, there is extra-large quantity of fluid, buoyant force of which was, however, never given prominence. The buoyant force of denser fluid apparently reduces gravitational load, thereby enhances the distance of separation between two gravitating bodies. Biomass mostly remains under neutral buoyant condition. As such, under a closed surface within a membranous boundary, biomass can become an independent gravitating body and can interact with other gravitational bodies, as per general astrophysical principles of gravitation. Under such a state of affairs, astrophysical principles can be scaled down or compared to a biological micro world on the principle of equivalence.
So in this review, we would examine aspects of commonness in the various phenomena of biological micro-world with some astrophysical events and in support of evidences and reasoning.

\section{Unspecified role of extra-large quantity of fluids in living}

The presence of extra-ordinary quantity of fluids in living bodies is one of the most poorly understood aspects in life science. On average, the body of an adult human contains $60 \%$ water. Most of the water in the human body is contained inside cells. For example, a 70$\mathrm{kg}$ man is made up of about 42 liters of total water- 28 liters are intracellular water; 14 liters are found in extracellular fluid of which 3 liters are blood plasma, 1 liter is the transcellular fluid (cerebro-spinal fluid, ocular, pleural, peritoneal and synovial fluids), 10 liters is the interstitial fluid (including lymph), which is an aqueous medium surrounding cells. Also, all our vital organs contain different amounts of water: the brain, the lungs, the heart, the liver and the kidneys contain a large quantity of water - between 65 to $85 \%$ depending on the organ, while bones contain less water (but still 31\%). The most interesting fact is that whatever large quantity of fluid present, their accumulation generally varies according to age, context, etc. For instance, the body of a newborn is composed of more water $(75 \%)$ than that of an elderly person (50\%), who has more accretion mass than newborn. The density of water and fat varies under equal volume. At high temperature, fats and oils are less dense than water. In living organisms, there is a certain relation. The more muscular a body is, the more water it contains. Conversely, the more fat in the body, the less water the body contains - as body fat has little water. In case of the fetus, it follows a distinct curve. For instance, 


\section{International Journal of Advanced Multidisciplinary Scientific Research (IJAMSR) ISSN:2581-4281}

at 10 weeks gestation, amniotic fluid is 10 to 20 milliliters of volume, at 16 weeks gestation $\sim 250$ milliliters, at 33 weeks gestation $\sim 800$ milliliters, at 3839 weeks to reach a plateau of $\sim 1000$ milliliters, and finally decreases at 40 weeks to $~ 800$ milliliters. In fact, the total amount of water in the human body is found in three main locations: within cells (two-thirds of the water), in the space between cells and in blood (onethird of the water). The importance of such large volume of water, gas and that too in the central location in living bodies has not yet suitably defined in life science. Fluids don't only play a role for transportation, but it buoyant property, including gaseous pressure bounded in closed surface in the living organisms work as astrophysical seclusion from the external stronger gravitation field, making planet within planet situations for living organisms, similar to Moon remains secluded from the gravitational field of the Earth or Sun.

\section{Physical role of fluid in living bodies}

An egg floats on saline water, on working against earth's gravity, due to buoyant force. Buoyancy acts against the force of gravity and so makes objects seem lighter with respect to gravity. At microgravity, everything floats in the absence of gravitational attraction. These are phenomena of attraction or lack of attraction of mutual gravity. Similarly an intravenous drip of 'isotonic' saline not only corrects disturbances in the water and electrolytic balance, but also provides buoyant force against the gravitational weight [4]. In this matter, let us discuss the physical role of water in relation to living bodies in little details.
Floating mechanism of aquatic living mass for survival

It is believed that the evolution of life started from aquatic medium. Increase or decrease in water temperature; density and dynamic as well as the kinematic viscosity of the fluids are usually affected. Higher dynamic viscosity represents the "stickiness" of fluids. Effects of mass towards kinematic viscosity can be eliminated through dividing dynamic viscosity by density. It is easier to swim (or move blood) in warm water, but it's harder to float. Temperature driven changes of density of water have profound impacts on aquatic organisms.

Seclusion on reducing gravitational drag force under neutral buoyancy

Amniotic fluid index (AFI) is considered as a part of the undefined biophysical profile. Through ultrasonograph, deepest, unobstructed, vertical length is measured. Proper role of AFI remains elusive in health science. Similarly, cytoplasm is composed mainly of water and also contains enzymes, salts, organelles, and various organic molecules. Spaargaren [5] coined the term 'metabolically inert infrastructure' (MII) to describe liquids in the cell. Let us redefine the metabolically inert infrastructure as follows. For instance, in unicellular organisms, cell's environment- viz. a substratum beneath it, a liquid medium around it and neighboring cells beside it; whereas in multicellular organisms, aggregation of cells and tissues with sufficient intra and extra-cellular matrix in totality affect 'life' (Illustration 1). 


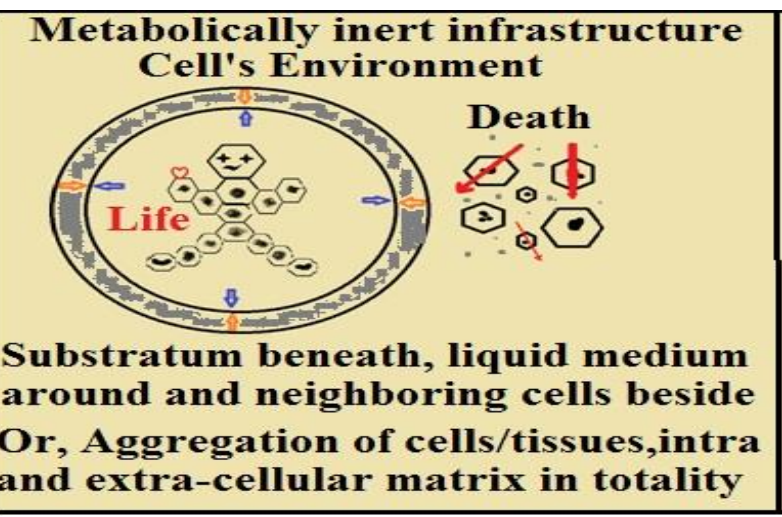

Illustration 1: Metabolically Inert Infrastructure includes the gravitational environment.

The mucilaginous jelly surrounding the embryo, or adequate depth of liquid media for multiplication of green algae, cultural media for bacteria, living host for viruses, plasmid or bacteriophage for transfer of a gene from one chromosome to other arts are some of the examples of undefined biophysical profiles. Without Metabolically Inert Infrastructure support death occurs. It is presumed that MII or 'medium' play the role of 'astrophysical distance', due to co-moving nonaccelerated position of the metabolically inert infrastructure (MII) that are relatively stationary or at constant velocity, or non-aligned or acting in the opposite direction of the energized accelerated self gravitating biomass or of the steady state supporting inertial reference frame at the specific point of time.

\section{Property of fluids to avert gravity's central pull}

Water has a high surface tension due to its adhesive and elastic property and tends to aggregate in drops on averting gravity's central pull. Thus, under a closed surface, fluid has the capacity to avert gravity's central pull and to provide desired thrust for maintaining distance in terms of astrophysical seclusion. The gaseous pressure works in tandem with fluid pressure. In animal cells, for instance, cytosol (fluids) works against central pull of self and mutual gravity, as shown in the freebody diagrams (Illustration 2). In the process, there was scope to understand transmission of fluid and gas pressure to increase in mass conforming Pascal's Law. Thus, with such fluids, gas within an enclosure or 'metabolically inert infrastructure', there would be an apparent seclusion of biomass or reduction of external gravitational pressure that work as "astrophysical distance on seclusion". Such closed surface would be like 'planet within planet' and would be of Gaussian in nature. Physical events within it can proceed in vitro independently.

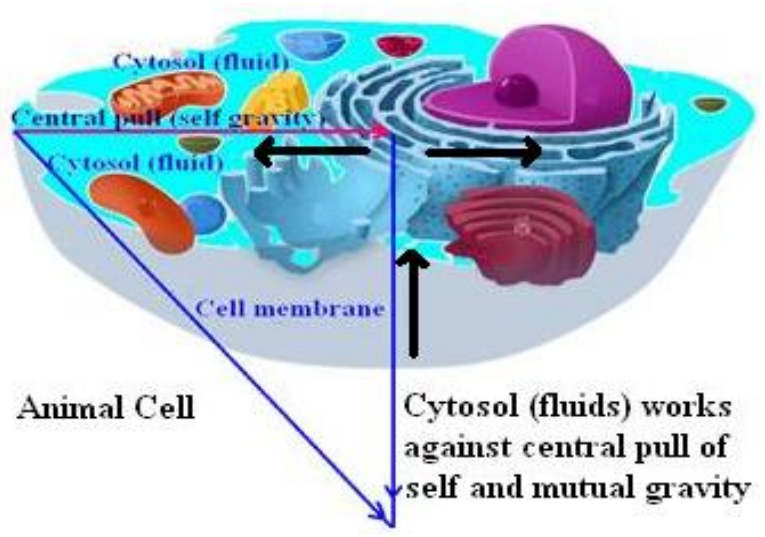

Illustration 2. Cytosol (fluid) causes substantial seclusion of organelles from the external gravitational field.

\section{Barrier mechanisms in Interstitium}

Organs which are necessary building blocks of organisms are collections of tissues with similar functions. Many organs coexist in the organ systems of plant and animal. In March 2018, an article was published in Scientific Reports titled 'Structure and 


\section{International Journal of Advanced Multidisciplinary Scientific Research (IJAMSR) ISSN:2581-4281}

Distribution of an Unrecognized Interstitium in Human Tissues'. Researchers Neil Theise \& his colleagues had discovered a previously unknown organ of reticular pattern in the human body. It comprised of a complex network of fluid-filled strong and flexible cavities. Such interstitium have spaces between cells, dynamically compressible and distensible sinuses through which interstitial fluid flows around the body. This is the largest by volume among all 80 organs. They observed similar structures in numerous tissues that are subjected to intermittent or rhythmic compression, including the submucosae of the entire gastrointestinal tract and urinary bladder, the dermis, the peri-bronchial and peri-arterial soft tissues. Interstitum is said to be distributed in the entire fascia, or as 'thin' mesh of tissue separating every muscle and all the tissue around every vein and artery, from largest to smallest. Among the major organ systems in the human body, circulatory, respiratory, digestive, excretory, nervous and endocrine systems are mostly located around central or core portion from the angle of fetus or birth position. Stomach for example, is positioned the middle and brain in the farthest region. It is said that the stomach is smarter than brain as there is more neurons in the gut than in the nervous system or brain. The built up of large network of fluid filled "interstitium" with tiny compartments work as if an amour jacket for the organ. It is presumed that viscosity of the fluid inside tiny compartment increases with applied external forces. As gravitational forces are maximum at the center of the tiny viscous fluid compartments, viscosity would rise next to central particles, which might form a barrier against external penetrating factors. As such hardness, impact of any compression and distention, swollen, enlargement will not be felt.

\section{Reduced level of fluid stop metabolism}

Studies in the brine shrimp showed that reducing the amount of water in a cell below $80 \%$ of the normal level inhibits metabolism, with this decreasing progressively as the cell dries out and all metabolism halting at a water level about $30 \%$ of normal. From the perspectives of gravitation an explanation to this observed phenomenon could be possible. With an inadequate depth of supporting fluids, macromolecular mass in the interior lost their gravitational seclusion - metabolic kinetic energy gets over-powered by potential gravitational energy (Illustration 3).

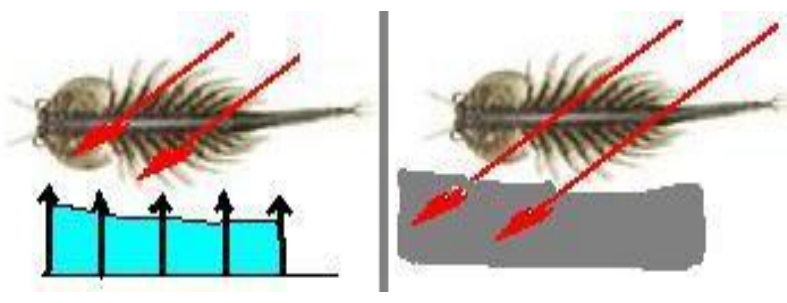

Illustration 3: Fluids cause substantial seclusion Reducing its level inhibit metabolism.

\section{Mass remains same but weight gets reduced}

A normal weight of a human child at birth is say 3200 gm on earth but at moon its weight would be $531 \mathrm{gm}$. The actual mass of the human brain is about 1400 grams; however, the net weight of the brain suspended in the cerebro-spinal fluid (CSF) is equivalent to a mass of 25 grams. i.e. what is $56 \mathrm{gm}$ in human body will appear to be 1 gram only. Thus, under neutral buoyant condition a 


\section{International Journal of Advanced Multidisciplinary Scientific Research (IJAMSR) ISSN:2581-4281}

body experiences a non-zero net force due to gravitational acceleration. Junwu $\mathrm{Mu}$ et al [6] made in vivo quantification of embryonic and placental growth during gestation in mice using micro-ultrasound and pairwise comparisons of in utero and ex utero measurements. They reported that when gestational age of mice reaches 16.5 days, the non-invasive predictive body weight remains to $0.792 \mathrm{gm}$ on average. The crown-rump length (CRL) and abdominal circumference (AC) was reported to be the function of gestational age (Illustration 4). The CRL and AC remain to be 16.22 $\mathrm{mm}$ and $23.4 \mathrm{~mm}$, respectively at that growth stage of mice. The average radius of the foetus can thus be considered to be roughly $9.9 \mathrm{~mm}$.
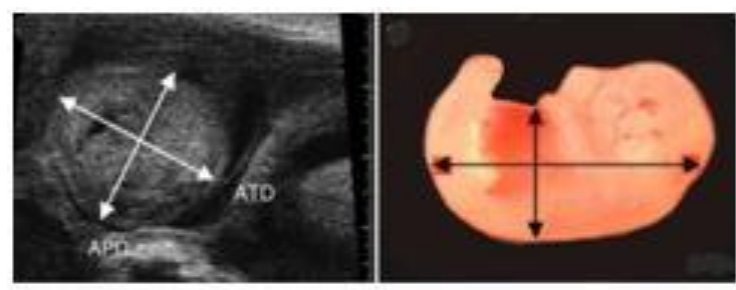

1. Illustration 4: In utero and ex utero measurements of foetus.

Magnitude of acceleration due to gravity under partial detached environment

Let us extend theoretically the foetal weight floating over amniotic fluid. Ignoring difference in the value of neutral buoyancy in cerebrospinal and amniotic fluids, due to differential presence of salt and other matters at particular location, the neutral buoyant weight of mice embryo of $0.792 \mathrm{gm}$ would appear to be $0.014 \mathrm{gm}$. The acceleration due to gravity on earth is about $9.8 \mathrm{~m} / \mathrm{s}^{2}$, whereas at the moon it is $1.62 \mathrm{~m} / \mathrm{s}^{2}$. However, if we calculate acceleration due to self gravity in 0.792 gram at 16 days of gestational age of mice with radius 9.9 $\mathrm{mm}$, separated by neutral buoyant force, as provided by Junwu Mu et al [6], using standard formula $\mathrm{g}(\mathrm{s})=$ $\mathrm{GM} / \mathrm{R}^{\wedge 2}$, it comes to be about $5 \times 10^{\wedge-9} \mathrm{~m} / \mathrm{s}^{\wedge}$. That is the free fall acceleration to the tune of 5 nanometers per second square in a massive body of the planet may be negligible, but in an isolated living mass at a distance of 9.9 picometers $\left(9.9 \times 10^{\wedge-12}\right.$ meter), acceleration of 5 $\mathrm{mm} / \mathrm{s}^{\wedge 2}$ is quite a significant force. Mass would have experienced a force of $7.70^{\wedge-8} \mathrm{~N}$ downwards due to gravity, had it been outside the fluid. But under neutral buoyant condition, mass would experience an upward resistance force/ upward force of the water of $0.14^{\wedge-8} \mathrm{~N}$. This upward force would be greater than the downward force of planetary or external gravity (Illustration 5).

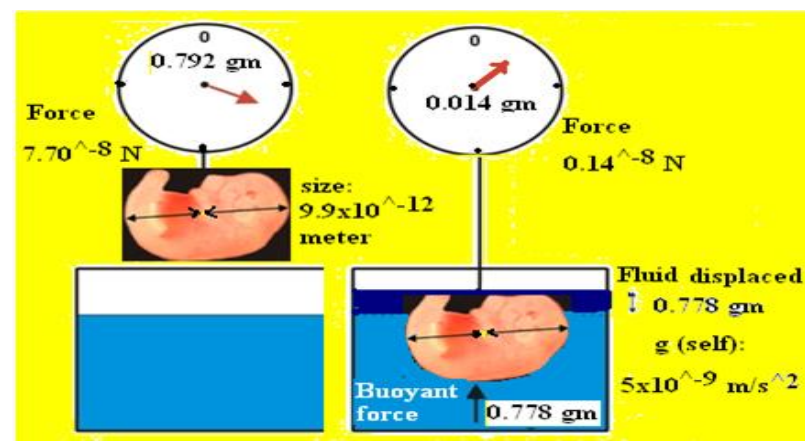

Illustration 5. Substantial seclusion of mass through weight reduction under fluid.

Gravitation induced mechanical movement of macromolecules

External fluid stresses, internal driving moments, and passive elastic resistance are generally considered as the primary cause of rafting of macromolecules over fluids. Pressure exerted anywhere in a confined incompressible fluid is transmitted equally in all directions throughout 


\section{International Journal of Advanced Multidisciplinary Scientific Research (IJAMSR) ISSN:2581-4281}

the fluid such that the pressure variations (initial differences) remain the same, as per Pascal's law or the principle of transmission of fluid-pressure. Gravity is an all-time force - slow but steady. Omnipresent but slow moving gravitation works similar to tortoise of the popular story 'The Hare and The Tortoise'. Unless variation in gravitational forces gets accounted, surface tension and hydrodynamic forces would appear to remain prominent. Say, if there is no resistance, equal and opposite bounding force, there would not have been an unbalanced pressure to exert. Our feet move due to the action of unbalanced force over the ground out of resistance or friction. Compressive action is needed to create unbalance pressure. Elastic force cannot be a substitute for gravitational force.

Assembly and heat production in astronomical objects

The stages of formation of planet are depicted (Illustration 6) to show how homogenous low density protoplanet after initial accretion becomes dense and differentiated planet on being exposed to gravitational contraction [7]. Under astrophysical principles, after initial accretion, planets are formed from homogenous low density protoplanet and become dense and differentiated planet on being exposed to gravitational contraction whereby size is reduced, density of accreted materials gets increased.

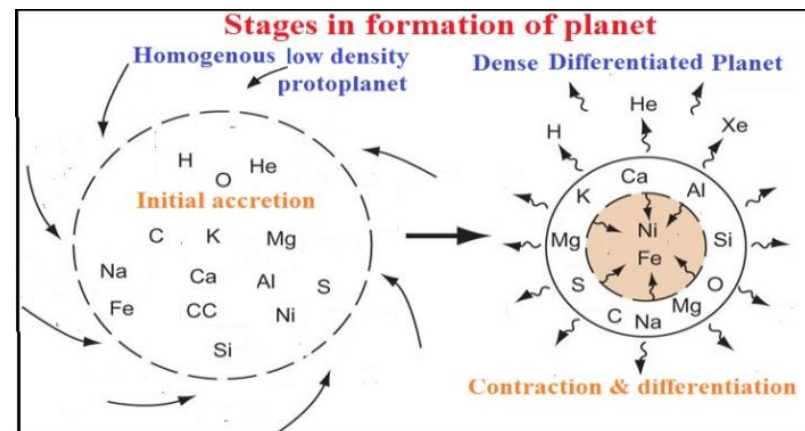

Illustration 6: Stages in formation in planet- initial accretion, contraction and differentiation.

In the core, gravitational compression produces heat. Gravitational pressure is then balanced by the outward thermal pressure from fusion reactions, temporarily halting gravitational compression. Equilibrium is reached when the compression is balanced by the pressure gradient. This pressure gradient is in the opposite direction due to the strength of the material, at which point gravitational compression ceases. Surface of star or planet gets cool. The cooling causes the pressure to drop and a frost line or ice line is formed. As a result the star or planet further shrinks. This compression, in turn, heats the core of the star/planet. So, alternate heating and cooling down or 'rhythmic' phenomena in growth is natural in all gravitating bodies.

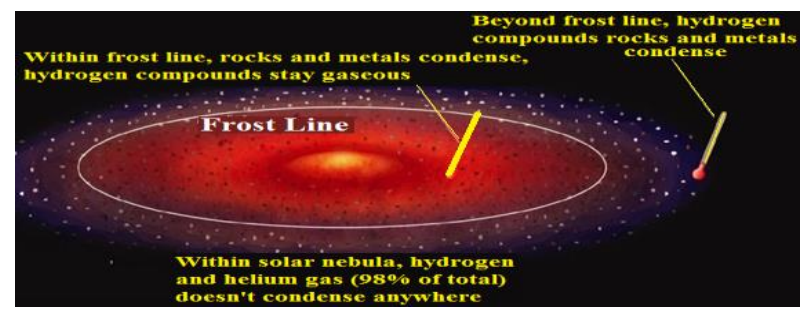

llustration 7: Formation of heat in core due to gravitational compression.

On balance of thermal pressure, surface gets cooled; frost line forms due to an opposite pressure gradient that leads to alternate heating and cooling down or 'rhythmic' phenomena in growth in store. 


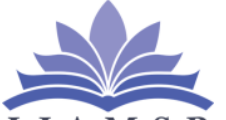

I J A M S R

\section{International Journal of Advanced Multidisciplinary Scientific Research (IJAMSR) ISSN:2581-4281}

What could produce heat in the human body?

What could produce heat in the human body? Constitution and physical environment of biological micro-world is completely different from planet formation. But the phenomena can be compared with the growth, development, differentiation, death in life science. Endothermic (warm-blooded) organisms create most of their heat via metabolic processes, but ectotherms (cold blooded) hires external sources of temperature to regulate their body temperatures. In human, hypothalamus (sensor) in the brain is not the heat producing center but only controls internal thermoregulations through neural feedback mechanisms. Radiation, perspiration, conduction and convection are the heat transfer mechanisms. Interestingly core body temperature lies in the central region liver, kidney, heart and parts of the brain under circular initial fetus position. Coldest part is at the periphery at the toe, foot, hand (Illustration 8). The molecules undergo elastic collisions. The faster the molecule collides, the hotter the surface would be. Thus the kinetic energy of molecules and kinetic energy of movement of sliding objects causes heating and cooling phenomena. Thus, it can be said that generation of heat in the human body is due to elastic collision of the molecules and kinetic energy of the sliding object, triggered by potential gravitational compression energy.

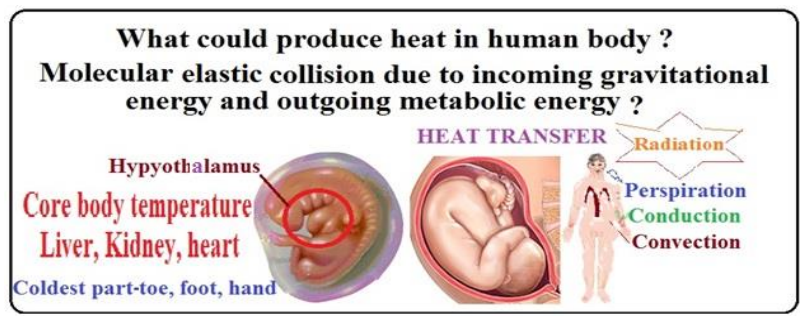

Illustration 8: Core body temperature in the central region during fetus stage; with heat transfer mechanisms - coldest parts lie in peripheral region- toe, foot, hand.
Homogeneous and heterogeneous accretion in planet

\section{formation}

In astrophysics, accretion is the accumulation of particles into a massive object by gravitationally attracting more matter, typically gaseous matter, in an accretion disk.

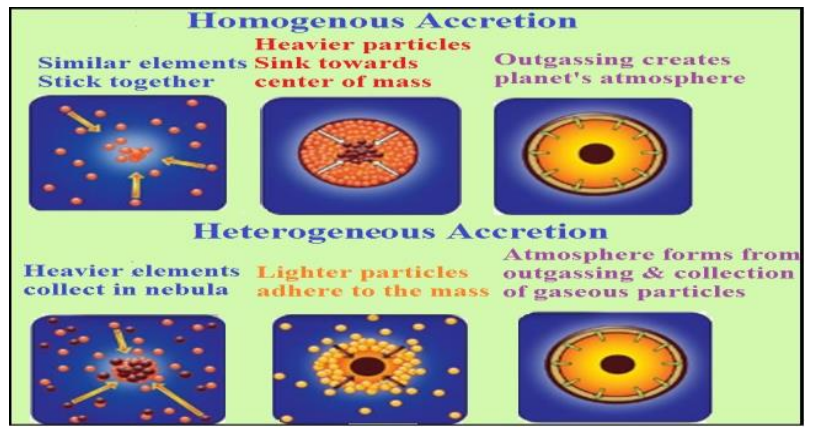

Illustration 9: Homogenous and heterogeneous accretion phenomena in astrophysics where similar elements stick together, heavier particles sink towards the center of mass, lighter particles adhere to the mass.

Galaxies, stars, and planets are formed by accretion processes. Homogenous accretion [7] is a phenomenon where similar elements stick together, heavier particles sink towards the center of mass and outgassing creates planet's atmosphere. Heterogeneous accretion means heavier elements collected in the nebula (center) and lighter particles adhere to the mass and subsequent stages of formation of atmosphere [8, 9] (Illustration 9). In a biological micro - world, though many little understood phenomena can be explained through homogenous and heterogenous accretion, but in the absence of proper viewing angle, these are often summed up as 'gift of nature'. 


\section{International Journal of Advanced Multidisciplinary Scientific Research (IJAMSR) ISSN:2581-4281}

Gravitation induced localization based on molar mass, density and temperature

Among the various macromolecules, as available in eukaryotes cells, nucleic acids have highest accretion of molar mass (say, 1000 - 5,000,000 g/mol) as well as density. Naturally, under gravitational (self) environment, nucleic acids would remain in the core position on the principle 'higher the mass and densityhigher would be the attractive force of self gravity'. Whole genome in the three-dimensional folded structure of DNA, if fully laid out would be around 6 feet long, but these fit into the nuclei of each of cells in the human body. Proteins are intermediate in accretion of molar mass (say, $75-180 \mathrm{~g} / \mathrm{Mol}$ ) as well as higher density (say, $1.1-1.4 \mathrm{~g} / \mathrm{ml}$ ), compared to the molar mass of water molecule of only $18 \mathrm{~g} / \mathrm{Mol}$. So it remains traditionally in the cytosol away from dynamic nucleus. Fats and lipids, though of comparable accretion of molar mass (say, $88-280 \mathrm{~g} / \mathrm{Mol}$ ) as that of proteins, but their accretions consist of the lowest density (say, $0.8-0.9$ $\mathrm{g} / \mathrm{ml}$ ). Hence, as per principle 'lesser the density of accreted material, there would be delay in gravitational attraction to the center'. In 'central dogma of molecular biology', it is interesting to note that replication of DNA (DNA - > DNA polymerase) and transcription of DNA to RNA (DNA - > RNA polymerase) traditionally occurs in the dynamic nucleus and translation of RNA to Protein (Ribosome) occurs in the cytoplasm away from the dynamic nucleus in the eukaryotic cell. Various exceptions for dynamic change of position with time were deliberately omitted to avoid detraction of attention from focus of the topic. Molar mass of nucleic acid (DNA, RNA) is higher than protein including ribosome. Ribosomes are large complexes of RNA and protein. Ribosomes are composed of two complex subunits, each of which includes rRNA and protein components.
Volume of nucleic acid is comparatively less than protein. Therefore the potential energy of gravitation could move dynamic nucleic acid to the central position. Thus macromolecules find position according to individual molar mass, density, variation in temperature and characteristic solubility (Illustration 10).

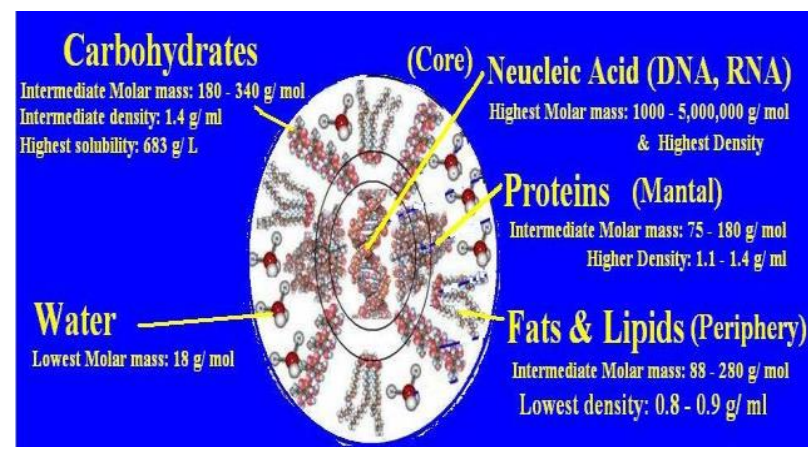

Illustration 10. Macromolecules find position according to individual molar mass, density, variation in temperature and characteristic solubility under neutral buoyant conditions.

Localization of Endoplasmic reticulum with or without ribosomes

Rough Endoplasmic Reticulum (RER) is coated with Ribosome. Ribosome is of higher molar mass than materials in the endoplasmic reticulum. Rough Endoplasmic reticulum (RER) is found . 


\section{International Journal of Advanced Multidisciplinary Scientific Research (IJAMSR) ISSN:2581-4281}

\section{Localization of Endoplasmic reticulum with or without ribosomes from dynamic core of self-gravitating living cell}

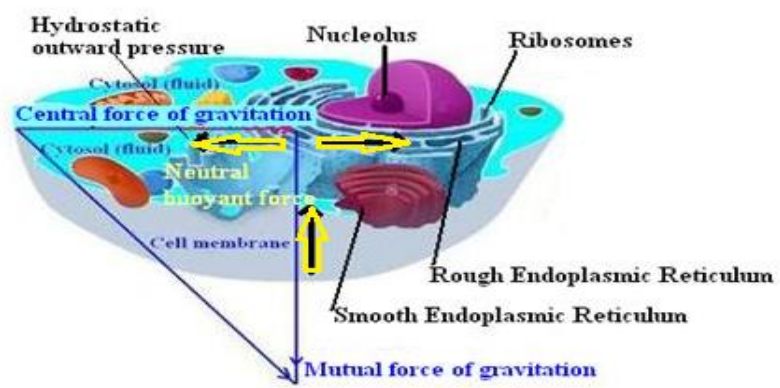

Illustration 11: Rough Endoplasmic reticulum (RER) studded with ribosomes is found to be distributed throughout the cell, but its density is higher near the dynamic nucleus or core.

On the other hand, Smooth Endoplasmic reticulum (SER), not studded with ribosomes, remains lighter and is found away from the dynamic core towards the periphery. SER is associated with comparatively less dense fats. The presence of higher weight ribosome nearer to the dynamic nucleus and association of lesser density fat away from the dynamic nucleus within a living cell (Illustration 11), thus, seems to obey the fundamental principle of universal gravitation within a eukaryotic cell.

Assembly and localization of fats, oils and lipids on heat and heat loss

Assembly and localization of fats, oils and lipids is primarily dependent on temperature and their physical state. At room temperature, fats are solid whereas oils are liquid. Among lipids, triglycerides are found in storage form; phospholipids being polar and non-polar, from cell membrane; lipoprotein work as carriers for fats in the blood. Both fats and oils are molecules shaped like a capital E with a glycerol spine and arms made of fatty acid chains. In animals, the carbon atoms in the fatty acids are saturated with hydrogen atoms. This allows them to lie straight and the molecules will pack neatly into a solid arrangement. In plants, missing hydrogen atoms cause kinks in the fatty acids. This reduces the amount of intermolecular bonding that can occur and keeps the molecules liquid at room temperature. The reason they have evolved this way is probably due to differences in plant and animal metabolisms. Lipids consisting of three molecules of fatty acids covalently bonded to one molecule of glycerol are termed triglycerides (or triacylglycerols), but are more commonly known as fats and oils. The division into fats and oils is arbitrary and depends on the physical state of these molecules at room temperature. Those molecules that are usually solid at room temperature (plant or animal) are termed fats, while those that are liquids at room temperature are termed oils. These latter are usually found in plants, but fish also store liquid forms of triglycerides (fish oils).

\section{Effect of temperature on accretion process}

The effect of temperature on accretion process, as happened in the planetary formation, has not yet studied in life science. Body temperature can increase as a result of exercise. A normal human core body temperature ranges from 36.5 degrees to 37.5 degrees Celsius. Depending upon the duration and intensity of exercise, temperature can easily reach above 40 degrees Celsius. During exercise, respiration releases energy in the muscles. When the body temperature is too high, the skin produces sweat, which evaporates from the surface 


\section{International Journal of Advanced Multidisciplinary Scientific Research (IJAMSR) ISSN:2581-4281}

of the skin. As it evaporates, it takes heat energy from the body. Oil is hydrophobic. Water is denser than oil. Since the ice is less dense than water and therefore less dense than oil, it floats at the top. As such distribution of oils and fats in disperse state are mostly temperature dependent phenomena and follow 'lower the density, slower will be the central attraction during accretion processes'. Among total cholesterol, there are LDL (low-density lipoprotein cholesterol, also called "bad" cholesterol) and also HDL (high-density lipoprotein cholesterol, also called "good" cholesterol). Regular exercise brings down a high LDL count. As exercise raises body temperature, low density lipoprotein can evade the process of accretion (Illustration 12).

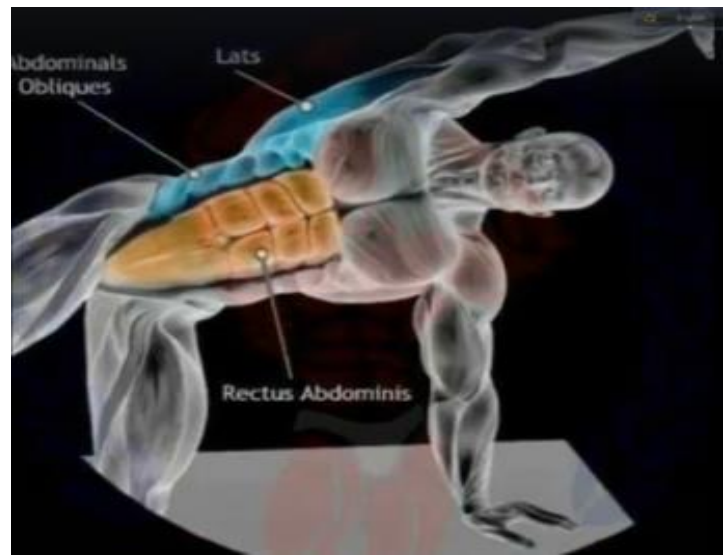

Illustration 12: Low density lipoprotein (fat) can evade process of accretion on exercise with rise in body temperature.

\section{Localization of carbohydrates}

Molar mass of carbohydrates is $180-340 \mathrm{~g} / \mathrm{mol}$ and their density is $1.4 \mathrm{~g} / \mathrm{ml}$. Monomer carbohydrates undergo polymerization and develop into long chained polysaccharides, such as cellulose, cellobioses, starch and glycogen (animal starch). Cellulose is made up of glucose units linked by $\beta 1>4$ linkages and it is an important component of the cell wall. Similarly starch is also a polymer of $\alpha 1-4$ linked glucose units and it is the main source of energy for all living cells. The most striking feature of monomer carbohydrates is that these posses highest solubility (683 g/ L) gets miscible with protoplasm, due to which, in general, carbohydrates cannot maintain its distinct location in a gravitational field, as do nucleic acid, proteins, fats and lipids. The protoplasm is granular colloidal in nature, because many macromolecules, tiny organelles are suspended in it, also exhibits sol and gel properties. Gutt [10] expressed that granular system exhibit duel properties - in a gravitational field, it may have a self-bounding free surface but also conform to the shape of the bounding wall, obeying continuum theories of fluid mechanics. As a compacted granular system, it can support shear stress in the absence of a shearing velocity. Application of granular physics is still at a nascent stage of study in life science. There is need for in-depth study on application of granular physics in life science to advance further on carbohydrate and other macromolecular movements.

\section{Ultracentrifugation as inverse process of central action of gravitation}

Under laboratory practices for separation of biological particles, centrifugation is adopted as common routine practice. Swedish chemist Theodor Svedberg [11] in 1925 developed the ultracentrifuge and won the 1926 Nobel Prize in chemistry, through which sub cellular materials, cells, large macromolecules like nucleic acids, proteins, ribosomes now separate from a solution. Depending on rotor speed of centrifugation (in anticlockwise direction) and the viscosity of the medium, various forces like centrifugal, inertial, gravitational, 


\section{International Journal of Advanced Multidisciplinary Scientific Research (IJAMSR) ISSN:2581-4281}

buoyant and frictional forces work between particles and solutions. Centrifugal acceleration is given as $\mathrm{r}^{\wedge}{ }^{\wedge}$; where $r$ is the radial distance from the rotation axis and $\omega$ is the angular velocity in radians per second. Sedimentation coefficients, expressed using Svedberg unit (symbol S) depends on mass, density, size, and shape. It is the ratio of the speed of a substance in a centrifuge to its acceleration in comparable units. When an area is bigger, say, a folded paper, if allowed to drop would reach its terminal velocity higher than the velocity of the same unfolded paper, as the area of the former would be larger and the friction force acting on it would be higher. In centrifugation, objects with larger surface area will travel at a slower terminal velocity. Here question lies, why Svedberg chooses centrifugation as a process of separation of biomaterials and not any chemical or other physical methods to separate in dispersed colloidal systems? In gravitational terms, 'centrifugation of any mass is an inverse process of central attraction'. The Svedberg values basically tell us about the comparative molecular weight and shape of biomaterials under ex vivo condition. Let us discuss the whole issue in details for understanding reality behind the process.

\section{Rate of sedimentation after centrifugation} corresponds to in vitro acceleration towards accretion

Bacteria and eukaryotes have ribosomes that are structurally different. Bacteria have so-called $70 \mathrm{~S}$ ribosomes and eukaryotes have $80 \mathrm{~S}$ ribosomes. However, it is admitted fact that the ribosomes and their sub-particles are heterogeneous accretion. They are named according to their sedimentation characteristics during centrifugation. The ribosomes subunits are named 60S and 40S for their "size" in Svedberg units for eukaryotes. In prokaryotes (bacteria), these are $30 \mathrm{~S}$ and 50S. These subunits are made up of three forms of rRNA: 16S, 23S, and 5S. For bacterial ribosomes, ultracentrifugation yields intact ribosomes (70S) as well as separate ribosomal subunits, the large subunit (50S) and the small subunit (30S). Within cells, ribosomes normally exist as a mixture of joined and separate subunits. The largest particles (whole ribosomes) sediment nears the bottom of the tube, whereas the smaller particles (separate 50S and 30S subunits) appear in upper fractions. Svedberg values are depicted as $1 \mathrm{x} \mathrm{g}$. The symbol $\mathrm{g}$ is considered as a relative centrifugal force; where relative means normalized to the acceleration due to gravity on earth $g=9.81 \mathrm{~m} / \mathrm{s}^{\wedge 2}$. One Svedberg (S) unit is $10^{\wedge-13}$. That is, a $1 \mathrm{~S}$ particle travels a distance of at the rate of $10^{\wedge-13} \mathrm{~m} \mathrm{~s}^{\wedge-1}$ or 0.1 picometers per second. Particles with higher values of $\mathrm{S}$ will travel proportionately faster, and increasing g force will also increase sedimentation rate. The two eukaryotic ribosomal subunits have sedimentation coefficients of 40 $\times 10^{\wedge}-13$ and $60 \times 10^{\wedge}-13$, are referred to as the $40 \mathrm{~S}$ and the $60 \mathrm{~S}$ ribosomal subunits. The molecular mass of the $40 \mathrm{~S}$ and $60 \mathrm{~S}$ particles are 1.5 and 3.0 million $\mathrm{g} / \mathrm{mol}$, respectively. Thus, the complete heterogeneous ribosome has a mass of approximately 4.5 million g/Mol.

Under the influence of an acceleration of a million gravities $\left(10^{\wedge} \mathrm{m} / \mathrm{s}^{\wedge}\right)$, a substance with a sedimentation coefficient of $80 \mathrm{~S}\left(80 \times 10^{\wedge-13} \mathrm{~s}\right)$ would travel at 80 micrometers per second $\left(80 \times 10^{\wedge-6} \mathrm{~m} / \mathrm{s}\right)$. Now, say, an $80 \mathrm{~S}$ ribosome at $100000 \mathrm{~g}$ centrifugation over the sucrose cushion buffer; the rate of sedimentation is equal to $10^{\wedge}-13 \times 10^{\wedge} \times 80 \mathrm{~m}$ per sec or $80 \times 10^{\wedge-8} \mathrm{~m} \mathrm{~s}^{\wedge-1}$. In order to travel, say, $10 \mathrm{~cm}\left(10^{\wedge-2} \mathrm{~m}\right)$, it would take $10^{\wedge-2} /$ $\left(80 \times 10^{\wedge}-8\right)$ s i.e. approximately 8.5 hours. It means that 
$80 \cdot 10^{\wedge-13} \mathrm{sec}$ is the time that ribosome would take to reach the terminal velocity in the fluid under ex vivo condition. Earlier, under in vitro situation, we have demonstrated that free fall acceleration to the tune of nanometer per Second Square in an isolated living mass of the picometer distance.

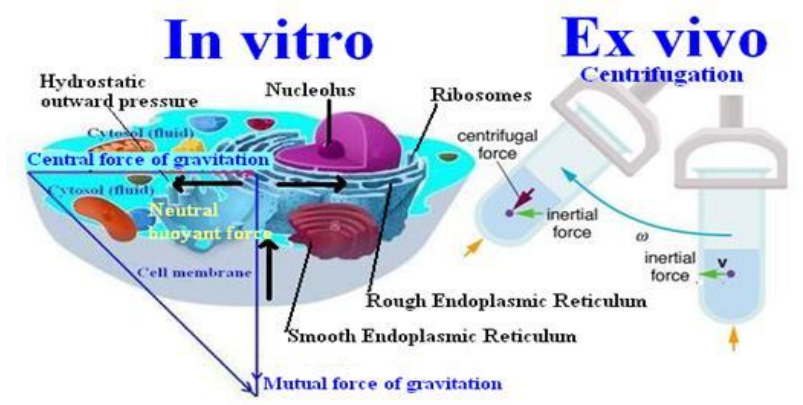

Illustration 13. Theodor Svedberg developed ultracentrifugation as a process of separation of bioparticles - inverse process of the central action of gravitation.

Under ex vivo situation, for moving $10 \mathrm{~cm}$ distance at a speed of 80 micrometers per second, $80 \mathrm{~S}$ ribosome is taking approximately 8 hours 30 minutes. It may be considered as approximate matching between in vitro and ex vivo observations, thereby indicating that both the process have their origin from the same sources, first one is on 'natural setting' and second one is an 'inverse or reverse of the natural setting'. Pre-centrifugation materials under in vitro situation are arranged as per density gradient influenced by the complex gravitation field 'within the cell'. Post-centrifugation order of succession depicts ex vivo sedimentation or setting as per external gravitational or mutual fields (Illustration 13). Centrifugation facilitates reversing the process for separation of organelles of losing its compression memory under ex vivo condition.

\section{Conclusions:}

Gravitation is slow but steady in its action, compared to electro-magnetic or electrostatic forces. Due to its slow rate of action, gravity could generally evade one's attention, especially amid an electrostatic field. Detection or undertaking quantitative measures of gravity is still a matter of challenge before physicists at the level of millimeter scale. So at this state of affairs, only way to establish is to apply abductive reasoning on comparing various circumstantial evidences. Few of such primary evidences are highlighted in the summary below:

a) Fluids constitute $50-90 \%$ of the total accredited biomass. Buoyant property of fluids bounded in closed surface in the living organisms work as astrophysical seclusion from the external stronger gravitation field, making planet within planet situations for living organisms, similar to Moon's internal processes remains secluded from the gravitational field of the Earth or Sun. Seclusion is reflected on reduction of 'Weight' under the same quantity of biomasses. Gravitational free fall acceleration, as estimated, to the tune of nanometer per second square in a massive body of the planet may be negligible, but in an isolated living mass at picometer distance, such acceleration is quite a significant force. Recently discovered network of interstitium with higher concentration in the middle region amidst empty space could be a phenomenon attached with fluid and gaseous distribution mechanisms in a gravitation environment. 


\section{International Journal of Advanced Multidisciplinary Scientific Research (IJAMSR) ISSN:2581-4281}

b) Homogenous accretion is a phenomenon in astrophysics where similar elements stick together, heavier particles sink towards the center of mass and out gassing creates planet's atmosphere. Heterogeneous accretion means heavier elements collected in the nebula (center) and lighter particles adhere to the mass and subsequent stages of formation of the atmosphere. The molecules undergo elastic collisions. The faster the molecule collides, the hotter the surface would be. Thus the kinetic energy of molecules and kinetic energy of movement of sliding objects causes heating and cooling phenomena and rhythmic pattern of growth both in biological and astrophysical worlds.

c) Under astrophysical principles, after initial accretion, planets are formed from homogenous low density protoplanet and become dense and differentiated planet on being exposed to gravitational contraction whereby size is reduced, density of accreted materials gets increased. In the core, gravitational compression produces heat. Gravitational pressure is then balanced by the outward thermal pressure from fusion reactions, temporarily halting gravitational compression. Equilibrium is reached when the compression is balanced by a pressure gradient. This pressure gradient is in the opposite direction due to the strength of the material, at which point gravitational compression ceases. Surface of star or planet gets cool. The cooling causes the pressure to drop and a frost line or ice line is formed. As a result the star or planet further shrinks. This compression, in turn, heats the core of the star/planet. So, alternate heating and cooling down or 'rhythmic' phenomena are natural in all gravitating bodies. Endothermic (warm-blooded) organisms create most of their heat via metabolic processes, but ectotherms (cold blooded) hires external sources of temperature to regulate their body temperatures. In human, hypothalamus (sensor) in the brain is not the heat producing center, but only controls internal thermoregulations through neural feedback mechanisms. Radiation, perspiration, conduction and convection are the heat transfer mechanisms. Interestingly core body temperature lies in the central region liver, kidney, heart and parts of the brain under circular initial fetus position. Coldest part is at the periphery at toe, foot, hand. Hence there is enough of similarity between biological microworld and astrophysical word.

d) There is strong evidence of mechanical movement of macromolecules induced by gravitation. Macromolecules find position according to individual molar mass, density, variation in temperature and characteristic solubility under neutral buoyant conditions. Molar mass of nucleic acid (DNA, RNA) is higher than protein including ribosome. Therefore potential energy of gravitation could drag dynamic nucleic acid to the central position. 


\section{International Journal of Advanced Multidisciplinary Scientific Research (IJAMSR) ISSN:2581-4281}

e) Assembly and localization of fats, oils and lipids is primarily dependent on temperature and their physical state. For instance, low density lipoprotein (fat) can evade the process of accretion on exercise with a rise in body temperature. Localization of carbohydrates, due to its highest solubility and capacity to get miscible cannot maintain its distinct location in a gravitational field, as do nucleic acid, proteins, fats and lipids. For instance, protoplasm is granular colloidal in nature, because many macromolecules, tiny organelles are suspended in it. Thus, as a granular system, it exhibits sol and gel properties and demonstrate duel properties in a gravitational field. It may have a self-bounding free surface and also conform to the shape of the bounding wall, obeying continuum theories of fluid mechanics. As a compacted granular system, it can support shear stress in the absence of a shearing velocity. Therefore carbohydrate distribution is regulated under granular physics, deserves further study.

f) Centrifugation is the standard bio-protocol in the laboratory to separate ribosomes and other biomaterials. Sedimentation coefficients, expressed using Svedberg unit depends on mass, density, size, and shape; and its values basically demonstrate the comparative molecular weight or gravitational mass and shape under ex vivo condition. Centrifugation process for separation of organelles facilitates inversion or reversing the natural setting on losing its compression memory of 'natural setting' under ex vivo condition. Pre- centrifugation materials under in vitro situation are arranged as per density gradient influenced by the complex gravitation field 'within the cell'. Post-centrifugation order of succession depicts ex vivo sedimentation or setting as per external gravitational or mutual fields. This is one of the strongest evidence that living in vitro biomaterials remains under gravitating pressure.

Deciphering further the mimesis of biological microworld with the astrophysical world of gravitation is carried over to the succeeding article on perspectives.

Conflict of Interest: There is no conflict of interest

\section{References:}

1. Bhattacharjee, I.R. (2013) Self Gravity: The Major Investigation Gap in Life Science. Lambert Academic Publishing ISBN 978-3-659-42698-8.

2. Bhattacharjee, IR. (2013) Self Gravity: The Major Investigation Gap in Life Science (Part I). WebmedCentral BIOPHYSICS;4(6):WMC004279

doi:10.9754/journal.wmc.2013.004279 \& Bhattacharjee, IR. (2013) Self Gravity: The Major Investigation Gap in Life Science (Part II). WebmedCentral BIOPHYSICS;4(6):WMC004296 doi: 10.9754/journal.wmc.2013.004296 \&

3. Bhattacharjee, I.R. (2017) Gravitation induced mechanical movement in cells. Jr for Research: Vol 03, $01: 24-31$ ISSN: 2395-7549

http://www.journal4research.org/articles/J4RV3I101 5.pdf

4. Bhattacharjee, I.R. (1988) Gravity Dictates LifeDeath and Biological Growth (also agricultural productivity) Introductory Concept: ISBN-13: 9788170193203.

5. Spaargaren, D.H. (1994) Metabolic rate and body size- A new view on the 'Surface Law' for basic metabolic rate. Acta Biotheoretica. 42, (4). 263-269. http://philpapers.org/rec/SPAMRA 
6. Junwu Mu, John C Slevin, Dawei Qu, Sarah McCormick, and S Lee Adamson: (2008) In vivo quantification of embryonic and placental growth during gestation in mice using microultrasound.Reprod Biol Endocrinol. 2008; 6: 34. Published online 2008 August 12. doi: $\quad$ 10.1186/1477-7827-6-34 PMCID: PMC2527569

7. Safronov V.S., Ruskol E.L. (1994) Formation and Evolution of Planets. In: Burke B.F., Rahe J.H., Roettger E.E. (eds) Planetary Systems: Formation, Evolution, and Detection. Springer, Dordrecht.

8. Rubie, D.C., Jacobson, S.A., Morbidelli, A., O'Brien, D.P, Young, E.D., Vries, J. de, Nimmo, F., Palme. H., Frost, D.J. (2015) Accretion and differentiation of the terrestrial planets with implications for the compositions of early-formed Solar System bodies and accretion of water. Icarus 248: 89-108.

9. Ian Morison (2008). Introduction to Astronomy and Cosmology. J Wiley \& Sons. p. 48. ISBN 0-47003333-9

10. Gutt, Gary Michael. The physics of granular systems. Ph.D. Dissertation, 1989 California Institute of Technology. http://resolver.caltech.edu/CaltechETD:etd05302007-081951

11. Pedersen KO. The development of Svedberg's ultracentrifuge. 1976 Jul; Biophys Chem. 5(1-2):318. PubMed PMID: 786403. 